\title{
POINCARÉ TYPE INEQUALITY FOR DIRICHLET SPACES AND APPLICATION TO THE UNIQUENESS SET
}

\author{
KARIM KELLAY*
}

\begin{abstract}
We give an extension of Poincaré's type capacitary inequality for Dirichlet spaces and provide an application to study the uniqueness sets on the unit circle for these spaces.
\end{abstract}

\section{Introduction}

Let $\mathrm{D}$ be the open unit disk in the complex plane and let $\mathrm{T}=\partial \mathrm{D}$ be the unit circle. For $0<\alpha \leq 1$, the Dirichlet space $\mathscr{D}_{\alpha}$ consists of all analytic functions $f$ defined on $\mathrm{D}$ such that

$$
\mathscr{D}_{\alpha}(f):=\int_{\mathrm{T}} \int_{\mathrm{T}} \frac{|f(z)-f(w)|^{2}}{|z-w|^{1+\alpha}} \frac{|d z|}{2 \pi} \frac{|d w|}{2 \pi}<\infty .
$$

The space $\mathscr{D}_{\alpha}$ is endowed with the norm

$$
\|f\|_{\alpha}^{2}:=|f(0)|^{2}+\mathscr{D}_{\alpha}(f) .
$$

By [7], this norm is comparable to

$$
\sum_{n \geq 0}|\widehat{f}(n)|^{2}(1+n)^{\alpha}
$$

The classical Dirichlet space $\mathscr{D}_{1}$ is a subspace of the Sobolev space $\mathrm{W}^{1,2}(\mathrm{D})$, defined as the completion of $\mathscr{C}^{1}$ (D) under the norm

$$
\|f\|^{2}=\left|\int_{\mathrm{D}} f(z) d \mathrm{~A}(z)\right|^{2}+\int_{\mathrm{D}}|\nabla f(z)|^{2} d \mathrm{~A}(z),
$$

* This work was partially supported by ANR Dynop.

Received 6 July 2009. 
where $d \mathrm{~A}(z)$ is a normalized Lebesgue measure. Note that the restriction of this norm to $\mathscr{D}_{1}$, becomes

$$
\|f\|^{2}=|f(0)|^{2}+\int_{\mathrm{D}}\left|f^{\prime}(z)\right|^{2} d \mathrm{~A}(z), \quad f \in \mathscr{D}_{1},
$$

which is equivalent to the norm of $\mathscr{D}_{1}$.

Given $f \in \mathrm{W}^{1,2}(\mathrm{D})$, we write $\mathrm{Z}(f)=\{z \in \mathrm{D}: f(z)=0\}$, the zero set of $f$ in $\mathrm{D}$. The Poincaré capacitary inequality in $\mathrm{W}^{1,2}(\mathrm{D})$ gives the precise asymptotic behavior of the constant in Poincaré's inequality [14], [19], [9], [1] (see also the paper [12] by Maz'ya and the references there). More precisely there exists a constant $c>0$ such that

$$
\int_{\mathrm{D}}|f(z)|^{2} d \mathrm{~A}(z) \leq \frac{c}{\mathrm{cap}_{2}(\mathrm{Z}(f))} \int_{\mathrm{D}}|\nabla f(z)|^{2} d \mathrm{~A}(z),
$$

for all $f \in \mathrm{W}^{1,2}(\mathrm{D}),\|\nabla f\|_{2} \neq 0$, where

$$
\operatorname{cap}_{2}(E)=\inf \left\{\int_{\mathrm{D}}|\nabla \varphi|^{2}: \varphi \in \mathrm{C}_{0}^{\infty}(\mathrm{D}), \varphi \geq 1 \text { on } E\right\}
$$

and $C_{0}^{\infty}(D)$ is the set of all infinitely differentiable functions of compact support in D. Our main result in this paper is to establish a Poincaré capacitary inequality for functions in the Dirichlet spaces with the zero set is contained in T (see Theorem 2.2). We provide a sufficient condition for a set to be uniqueness set for Dirichlet spaces (see Theorem 3.1).

Let $\mathrm{X}$ be some class of analytic functions in $\mathrm{D}$ and let $E$ be a subset of T. The set $E$ is said to be a uniqueness set for $\mathrm{X}$ if, for each $f \in \mathrm{X}$ such that $f^{*}(\zeta):=\lim _{r \rightarrow 1-} f(r \zeta)=0$ for all $\zeta \in E$, we have $f=0$.

It is clear that $\mathscr{D}_{\alpha}$ is contained in the Hardy space $\mathrm{H}^{2}$. So each function $f \in \mathscr{D}_{\alpha}$ has non-tangential limits a.e on T. It is known that every set $E \subset \mathrm{T}$ of positive Lebesgue measure is a uniqueness set for all functions of bounded type in $\mathrm{D}$ (and therefore, for $\mathrm{H}^{2}$ ). Carleson [4] proved that a closed set of Lebesgue measure zero $E \subset \mathrm{T}$ is a uniqueness set for the Lipschitz class if and only if $E$ is not a Carleson set $\left(\log \operatorname{dist}(\cdot, E) \notin \mathrm{L}^{1}(\mathrm{~T})\right)$. He also proved in the same paper that if $E$ is not a Carleson set under capacitary condition (in particular $E$ has a positive $C_{s}$-capacity for some $s>0$ ), then $E$ is a uniqueness set for the classical Dirichlet space. Khavin and Maz'ya [9] have proved that there exists a set of uniqueness of $C_{s}$-capacity zero for any $s>0$ for the classical Dirichlet space. The proof of Khavin and Maz'ya is based on Poincaré's inequality in the Sobolev space (1). However, the Khavin-Maz'ya Theorem does not allow to deduce the Carleson Theorem. Here, we give a generalization of Khavin-Mazya's result which works for $\mathscr{D}_{\alpha}$ spaces, $0<\alpha \leq 1$, and from 
it we deduce Carleson's result. Our proof is based on a local Poincaré type capacitary inequality in Dirichlet spaces (see Theorem 2.2).

\section{Poincaré's capacitary inequality}

\subsection{Capacity}

We begin with the definition of the classical capacity [5], [8]. We define the kernel on $\mathrm{T}$ by

$$
k_{\alpha}(\xi)= \begin{cases}|1-\zeta|^{-\alpha}, & 0<\alpha<1, \\ |\log | 1-\zeta||, & \alpha=0 .\end{cases}
$$

Given a probability measure $\mu$ on T, for $0 \leq \alpha<1$, we define its $\alpha$-energy by

$$
I_{\alpha}(\mu)=\iint k_{\alpha}(\zeta \bar{\xi}) d \mu(\xi) d \mu(\zeta) .
$$

Given a Borel subset $E$ of T, we denote by $\mathscr{P}(E)$ the set of all probability measures supported on a compact subset of $E$. We define its $C_{\alpha}$-capacity by

$$
C_{\alpha}(E)=1 / \inf \left\{I_{\alpha}(\mu): \mu \in \mathscr{P}(E)\right\} .
$$

If $\alpha=0, C_{0}$ is called the logarithmic capacity. Note that for a set $E \subset \mathrm{T}$, $C_{\alpha}(E)>0$ means that there exists a Borel positive finite measure $\mu$ supported by $E$ with finite energy

$$
\sum_{n \geq 1} \frac{|\widehat{\mu}(n)|^{2}}{n^{1-\alpha}}<\infty .
$$

Now we define the $\mathrm{L}^{2}$-capacity introduced by Meyers [13] see also [1], [2]. For $0<\alpha \leq 1$, the harmonic Dirichlet space $\mathscr{D}_{\alpha}(\mathrm{T})$ consists of all functions $f \in \mathrm{L}^{2}(\mathrm{~T})$ such that

$$
\mathscr{D}_{\alpha}(f)<\infty
$$

with the norm

$$
\|f\|_{\mathscr{D}_{\alpha}(\mathrm{T})}^{2}=\|f\|_{\mathrm{L}^{2}(\mathrm{~T})}^{2}+\mathscr{D}_{\alpha}(f) .
$$

This norm is comparable to

$$
\sum_{n \geq 0}|\widehat{f}(n)|^{2}(1+|n|)^{\alpha} .
$$

We have $\widehat{k_{1-\frac{\alpha}{2}}}(n) \sim|n|^{-\frac{\alpha}{2}}$ as $n \rightarrow \pm \infty$ and so $\left\|k_{1-\frac{\alpha}{2}} \star f\right\|_{\alpha}$ is comparable to $\|f\|_{\mathrm{L}^{2}(\mathrm{~T})}$ for all $f \in \mathrm{L}^{2}(\mathrm{~T})$. Hence

$$
\mathscr{D}_{\alpha}(\mathrm{T})=\left\{k_{1-\frac{\alpha}{2}} \star f: f \in \mathrm{L}^{2}(\mathrm{~T})\right\} .
$$


For any set $E \subset \mathrm{T}$ we define the $C_{\alpha, 2}$ capacity by

$$
C_{\alpha, 2}(E):=\inf \left\{\|f\|_{\mathrm{L}^{2}(\mathrm{~T})}^{2}: f \in \mathrm{L}^{2}(\mathrm{~T}), f \geq 0, k_{1-\frac{\alpha}{2} \star} \star f \geq 1 \text { on } E\right\} .
$$

This capacity is comparable to

$$
\inf \left\{\|f\|_{\mathscr{D}_{\alpha}(\mathrm{T})}^{2}: f \in \mathscr{D}_{\alpha}(\mathrm{T}), f \geq 0, f \geq 1 \text { on } E\right\} .
$$

Furthermore $C_{\alpha, 2}(E)$ is comparable to the classical capacity $C_{1-\alpha}$, where the implied constants depend only on $\alpha$, see [13] Theorem 14, [1] Theorem 2.5.5. We finally mention the results of Beurling [3] and Salem Zygmund [8], [5], [4] about the boundary behavior for the functions of the Dirichlet spaces: if $f \in \mathscr{D}_{\alpha}$, we write $f^{*}(\xi)=\lim _{r \rightarrow 1-} f(r \xi)$, then $f^{*}$ exists $C_{1-\alpha}$-q.e on T, that is

$$
C_{1-\alpha}\left(\left\{\zeta \in \mathrm{T}: f^{*}(\zeta) \text { does not exist }\right\}\right)=0 .
$$

Note that if $E$ is a closed set such that $C_{1-\alpha}(E)=0$, then there exists a function $f \in \mathscr{D}_{\alpha}$ with $f^{*}(\zeta)=0$ on $E$ (see [4]).

\subsection{Poincaré's capacitary inequality for the Dirichlet spaces}

Let $I, J$ be two open arcs of T and $f$ be a function. We set

$$
\mathscr{D}_{I, J, \alpha}(f)=\int_{I} \int_{J} \frac{|f(z)-f(w)|^{2}}{|z-w|^{1+\alpha}} \frac{|d z|}{2 \pi} \frac{|d w|}{2 \pi},
$$

and

$$
\mathscr{D}_{I, \alpha}(f)=\mathscr{D}_{I, I, \alpha}(f)
$$

We begin with a simple extension lemma.

LemMA 2.1. Let $0<\gamma<1$ and let $I=\left(e^{-i \theta}, e^{i \theta}\right)$ with $\theta<\gamma \pi / 2$. Let $f \in \mathscr{D}_{\alpha}$, then there exists a function $\widetilde{f}$ coincide with $f$ in I and such that

$$
\mathscr{D}_{J, \alpha}(\tilde{f}) \leq c \mathscr{D}_{I, \alpha}(f),
$$

where $J=\left(e^{-2 i \theta /(1+\gamma)}, e^{2 i \theta /(1+\gamma)}\right)$ and c an absolute constant.

Proof. Let $\tilde{f}$ be such that

$$
\tilde{f}\left(e^{i t}\right)= \begin{cases}f\left(e^{i t}\right) & e^{i t} \in I, \\ f\left(e^{i \frac{3 \theta-t}{2}}\right) & e^{i t} \in L:=\left(e^{i \theta}, e^{2 i \theta /(1+\gamma)}\right), \\ f\left(e^{-i \frac{3 \theta+t}{2}}\right) & e^{i t} \in R:=\left(e^{-2 i \theta /(1+\gamma)}, e^{-i \theta}\right) .\end{cases}
$$

We write

$$
\begin{aligned}
\mathscr{D}_{J, \alpha}(\tilde{f})=\mathscr{D}_{I, \alpha}(f)+\mathscr{D}_{L, \alpha} & (\tilde{f})+\mathscr{D}_{R, \alpha}(\tilde{f}) \\
& +2 \mathscr{D}_{I, L, \alpha}(\tilde{f})+2 \mathscr{D}_{I, R, \alpha}(\tilde{f})+2 \mathscr{D}_{L, R, \alpha}(\tilde{f}) .
\end{aligned}
$$


If $u, v \in\left(\frac{1+3 \gamma}{2(1+\gamma)} \theta, \theta\right)$, then $\pi>|2 u-2 v| \geq|u-v|$. By change of variable, we get

$$
\mathscr{D}_{L, \alpha}(\tilde{f})=4 \int_{\frac{1+3 \gamma}{2(1+\gamma)} \theta}^{\theta} \int_{\frac{1+3 \gamma}{2(1+\gamma)} \theta}^{\theta} \frac{\left|f\left(e^{i u}\right)-f\left(e^{i v}\right)\right|^{2}}{\left|e^{i(3 \theta-2 u)}-e^{i(3 \theta-2 v)}\right|^{1+\alpha}} \frac{d u}{2 \pi} \frac{d v}{2 \pi} \leq 4 \mathscr{D}_{I, \alpha}(f) .
$$

The same inequality holds for $\mathscr{D}_{R, \alpha}(\tilde{f})$.

If $u \in\left(\frac{1+3 \gamma}{2(1+\gamma)} \theta, \theta\right)$ and $t \in(-\theta, \theta)$, then $\pi>3 \theta-2 u-t \geq|u-t|$ and

$$
\mathscr{D}_{I, L, \alpha}(\tilde{f})=2 \int_{-\theta}^{\theta} \int_{\frac{1+3 \gamma}{2(1+\gamma)}}^{\theta} \frac{\left|f\left(e^{i t}\right)-f\left(e^{i u}\right)\right|^{2}}{\left|e^{i t}-e^{i(3 \theta-2 u)}\right|^{1+\alpha}} \frac{d v}{2 \pi} \frac{d t}{2 \pi} \leq 2 \mathscr{D}_{I, \alpha}(f) .
$$

The same inequality holds also for $\mathscr{D}_{I, R, \alpha}(\tilde{f})$.

If $u \in\left(\frac{1+3 \gamma}{2(1+\gamma)} \theta, \theta\right)$ and $v \in\left(-\theta,-\frac{1+3 \gamma}{2(1+\gamma)} \theta\right)$, then $\pi>(3 \theta-2 u)+(3 \theta+$ $2 v) \geq u-v$ and

$$
\begin{aligned}
\mathscr{D}_{L, R, \alpha}(\tilde{f}) & =4 \int_{\frac{1+3 \gamma}{2(1+\gamma)} \theta}^{\theta} \int_{-\theta}^{-\frac{1+3 \gamma}{2(1+\gamma)} \theta} \frac{\left|f\left(e^{i u}\right)-f\left(e^{i v}\right)\right|^{2}}{\left|e^{i(2 \theta-2 u)}-e^{-i(2 \theta+2 v)}\right|^{1+\alpha}} \frac{d v}{2 \pi} \frac{d u}{2 \pi} \\
& \leq 4 \mathscr{D}_{I, \alpha}(f) .
\end{aligned}
$$

Hence (2) is proved.

Given $E \subset \mathrm{T}$, we write $|E|$ for the Lebesgue measure of $E$. We can now state the main result of this section.

Theorem 2.2. Suppose that $0<\gamma<1$. Let $E \subset \mathrm{T}$ and $f \in \mathscr{D}_{\alpha}$ be such that $f^{*} \mid E=0$. Then, for any open arc $I \subset \mathrm{T}$ with $|I| \leq \gamma \pi$ and any $0<\beta \leq \alpha$,

$$
\left[\frac{1}{|I|} \int_{I}|f(\xi)||d \xi|\right]^{2} \leq \frac{c|I|^{\alpha-\beta}}{C_{\beta, 2}(E \cap I)} \mathscr{D}_{I, \alpha}(f),
$$

where $c$ is a constant depending only on $\beta$ and $\gamma$.

Proof. For simplicity, we will assume that $I=\left(e^{-i \theta}, e^{i \theta}\right)$ with $\theta<\gamma \pi / 2$. Let $J=\left(e^{-2 i \theta /(1+\gamma)}, e^{2 i \theta /(1+\gamma)}\right), \theta_{\gamma}=\frac{3+\gamma}{2(1+\gamma)} \theta$ the midpoint of $(\theta, 2 \theta /(1+\gamma))$ and $I_{\gamma}=\left(e^{-i \theta_{\gamma}}, e^{i \theta_{\gamma}}\right)$. Let $\phi$ be a positive function on T such that $\operatorname{supp} \phi=I_{\gamma}$, $\phi=1$ on $I$ and

$$
|\phi(z)-\phi(w)| \leq \frac{c_{\gamma}}{|J|}|z-w|, \quad z, w \in \mathrm{T} .
$$

where $c_{\gamma}$ is a constant depending only on $\gamma$. 
Now let $\tilde{f}$ be the function given in Lemma 2.1 and set

$$
F(z)=\phi(z)\left|1-\frac{|\tilde{f}(z)|}{m}\right|, \quad z \in \mathrm{T},
$$

with

$$
m:=\frac{1}{|J|} \int_{J}|\tilde{f}(\zeta)||d \zeta|
$$

Hence $F \geq 0, F_{\mid E \cap I}=1 C_{1-\alpha}$-q.p and thus $F_{\mid E \cap I}=1 C_{1-\beta}$-q.p, since if $C_{1-\alpha}(A)=0$, we have $C_{1-\beta}(A)=0$. Therefore,

$$
\begin{aligned}
C_{\beta, 2}(E \cap I) & \simeq \inf \left\{\|g\|_{\mathscr{D}_{\beta}(\mathrm{T})}^{2}: g \geq 0, g \geq 1 C_{\beta, 2} \text {-q.p on } E \cap I\right\} \\
& \leq c_{\beta}\|F\|_{\mathscr{D}_{\beta}(\mathrm{T})}^{2},
\end{aligned}
$$

where $c_{\beta}$ is a constant depending only on $\beta$.

In order to conclude, we estimate $\|F\|_{\mathscr{D}_{\beta}(\mathrm{T})}^{2}$. First,

$$
\begin{aligned}
\|F\|_{\mathscr{D}_{\beta}(\mathrm{T})}^{2}= & \int_{\mathrm{T}}|F(z)|^{2} \frac{|d z|}{2 \pi}+\int_{\mathrm{T}} \int_{\mathrm{T}} \frac{|F(z)-F(w)|^{2}}{|z-w|^{1+\beta}} \frac{|d z|}{2 \pi} \frac{|d w|}{2 \pi} \\
\leq & \left.\frac{1}{m^{2}} \int_{J}|m-| \tilde{f}(z)\right|^{2} \frac{|d z|}{2 \pi}+\int_{J} \int_{J} \frac{|F(z)-F(w)|^{2}}{|z-w|^{1+\beta}} \frac{|d z|}{2 \pi} \frac{|d w|}{2 \pi} \\
& \quad+\frac{2}{m^{2}} \int_{z \in \top \backslash J} \int_{w \in I_{\gamma}} \frac{\left.|m-| \tilde{f}(w)\right|^{2}}{|z-w|^{1+\beta}} \frac{|d z|}{2 \pi} \frac{|d w|}{2 \pi} \\
= & \frac{A}{2 \pi m^{2}}+\frac{B}{4 \pi^{2}}+\frac{C}{2 \pi^{2} m^{2}} .
\end{aligned}
$$

By (2),

$$
\begin{aligned}
A & :=\int_{J}|m-| \tilde{f}(z)||^{2}|d z|=\left.\frac{1}{|J|^{2}} \int_{J}\left|\int_{J}(|\tilde{f}(\zeta)|-|\tilde{f}(z)|)\right| d \zeta\right|^{2}|d z| \\
& \leq \frac{1}{|J|} \int_{J} \int_{J}|\tilde{f}(\zeta)-\tilde{f}(z)|^{2}|d \zeta||d z| \leq c_{1} \int_{J} \int_{J} \frac{|\widetilde{f}(\zeta)-\tilde{f}(z)|^{2}}{|\zeta-z|^{1+\beta}}|d \zeta||d z| \\
& \leq c_{1}|J|^{\alpha-\beta} \mathscr{D}_{J, \alpha}(\tilde{f}) \leq c_{2}|I|^{\alpha-\beta} \mathscr{D}_{I, \alpha}(f),
\end{aligned}
$$

for some constants $c_{1}, c_{2}$ independent of $\beta$ and $\gamma$. 
If $(z, w) \in J \times J$, then

$$
\begin{aligned}
& |F(z)-F(w)| \\
& \quad=\left|\phi(z)\left(\left|1-\frac{|\tilde{f}(z)|}{m}\right|-\left|1-\frac{|\tilde{f}(w)|}{m}\right|\right)+(\phi(z)-\phi(w))\right| 1-\frac{|\tilde{f}(w)|}{m}|| \\
& \quad \leq \frac{1}{m}|\tilde{f}(z)-\tilde{f}(w)|+\frac{c_{\gamma}}{m} \frac{|z-w|}{|J|}|m-| \tilde{f}(w)|| \\
& \quad \leq \frac{1}{m}|\tilde{f}(z)-\tilde{f}(w)|+\frac{c_{\gamma}}{m} \frac{|z-w|}{|J|^{2}} \int_{J}|\tilde{f}(\zeta)-\tilde{f}(w)||d \zeta| .
\end{aligned}
$$

So, by (2) again,

$$
\begin{aligned}
B:= & \int_{J} \int_{J} \frac{|F(z)-F(w)|^{2}}{|z-w|^{1+\beta}}|d z||d w| \\
\leq & \frac{2}{m^{2}} \int_{J} \int_{J} \frac{|\tilde{f}(z)-\tilde{f}(w)|^{2}}{|z-w|^{1+\beta}}|d z||d w| \\
& +\frac{2 c_{\gamma}^{2}}{m^{2}|J|^{4}} \int_{J} \int_{J}\left(\int_{J}|\tilde{f}(\zeta)-\tilde{f}(w)||d \zeta|\right)^{2}|z-w|^{1-\beta}|d w||d z| \\
\leq & \frac{2+2 c_{\gamma}^{2}}{m^{2}} \int_{J} \int_{J} \frac{|\tilde{f}(\zeta)-\tilde{f}(w)|^{2}}{|\zeta-w|^{1+\beta}}|d \zeta||d w| \leq \frac{c_{3}}{m^{2}}|I|^{\alpha-\beta} \mathscr{D}_{I, \alpha}(f),
\end{aligned}
$$

with $c_{3}$ is a constant depending only on $\gamma$.

Finally,

$$
\begin{aligned}
C & :=\int_{z \in \mathrm{T} \backslash J} \int_{w \in I_{\gamma}} \frac{\left.|m-| \tilde{f}(w)\right|^{2}}{|z-w|^{1+\beta}}|d z||d w| \\
& \leq \frac{c_{4}}{|J|^{1+\beta}} \int_{I_{\gamma}}|m-| \tilde{f}(w)||^{2}|d w| \\
& \leq \frac{c_{4}}{|J|^{2+\beta}} \int_{I_{\gamma}}\left|\int_{J}\right| \tilde{f}(\zeta)-\tilde{f}(w)|| d \zeta||^{2}|d w| \\
& \leq \frac{c_{4}}{|J|^{1+\beta}} \int_{I_{\gamma}} \int_{J}|\tilde{f}(\zeta)-\tilde{f}(w)|^{2}|d \zeta||d w| \\
& \leq c_{4} \iint_{J \times J} \frac{|\tilde{f}(\zeta)-\tilde{f}(w)|^{2}}{|\zeta-w|^{1+\beta}}|d \zeta||d w| \leq c_{5}|I|^{\alpha-\beta} \mathscr{D}_{I, \alpha}(f),
\end{aligned}
$$

with $c_{4}, c_{5}$ independent of $\gamma, \beta$. 
By (5), (6) and (7), we see that

$$
\|F\|_{\mathscr{D}_{\beta}(\mathrm{T})}^{2} \leq \frac{c_{6}}{m^{2}}|I|^{\alpha-\beta} \mathscr{D}_{I, \alpha}(f),
$$

with $c_{6}$ depending only on $\gamma$. Since

$$
m \asymp \frac{1}{|I|} \int_{I}|f(\xi)||d \xi|,
$$

combining (3) and (8), we get

$$
C_{\beta, 2}(E \cap I) \leq c\left[\frac{1}{|I|} \int_{I}|f(\xi)||d \xi|\right]^{-2}|I|^{\alpha-\beta} \mathscr{D}_{I, \alpha}(f),
$$

where $c$ depending only on $\beta$ and $\gamma$, and the proof is complete.

\section{Set of uniqueness for Dirichlet spaces}

A special case of the theorem ( $\beta=1$ in Theorem 3.1) was obtained by Khavin and Maz'ya [9] for the classical Dirichlet space $(\alpha=1)$. Here we give the generalization of their result in the Dirichlet spaces, including the classical case.

TheOREM 3.1. Let E be a Borel subset of T of Lebesgue measure zero. We assume that there exists a family of pairwise disjoint open arcs $\left(I_{n}\right)$ of $\mathrm{T}$ such that $E \subset \bigcup_{n} I_{n}$. Suppose that there exists $0<\beta \leq \alpha$ such that

$$
\sum_{n}\left|I_{n}\right| \log \frac{\left|I_{n}\right|^{1+\alpha-\beta}}{C_{1-\beta}\left(E \cap I_{n}\right)}=-\infty,
$$

then $E$ is a uniqueness set for $\mathscr{D}_{\alpha}$.

Proof. Since $|E|=0$, we can assume that there is $\gamma \in(0,1)$ such that $\sup _{n}\left|I_{n}\right| \leq \gamma \pi$. Let $f \in \mathscr{D}_{\alpha}$ be such that $f^{*} \mid E=0$. We set $\mathscr{I}=\sum_{n}\left|I_{n}\right|$. Since $\left(I_{n}\right)$ are disjoint, $C_{1-\beta}$ is comparable to $C_{\beta, 2}$. Then Theorem 2.2 and the Jensen inequality give

$$
\begin{aligned}
2 \int_{\cup I_{n}} & \log |f(\xi)| d \xi \mid \\
\leq & \sum_{n}\left|I_{n}\right| \log \left(\frac{1}{\left|I_{n}\right|} \int_{I_{n}}|f(\xi)| d \xi \mid\right)^{2} \\
\leq & \sum_{n}\left|I_{n}\right| \log \left(\frac{c\left|I_{n}\right|^{\alpha-\beta}}{C_{1-\beta}\left(E \cap I_{n}\right)} \mathscr{D}_{I_{n}, \alpha}(f)\right)
\end{aligned}
$$




$$
\begin{aligned}
& =\sum_{n}\left|I_{n}\right| \log \frac{\left|I_{n}\right|^{1+\alpha-\beta}}{C_{1-\beta}\left(E \cap I_{n}\right)}+\mathscr{I} \sum_{n} \frac{\left|I_{n}\right|}{\mathscr{I}} \log \left(c \mathscr{D}_{I_{n}, \alpha}(f)\right) \\
& \leq \sum_{n}\left|I_{n}\right| \log \frac{\left|I_{n}\right|^{1+\alpha-\beta}}{C_{1-\beta}\left(E \cap I_{n}\right)}+\mathscr{I} \log \left(\frac{c}{\mathscr{I}} \sum_{n} \mathscr{D}_{I_{n}, \alpha}(f)\right) \\
& \leq \sum_{n}\left|I_{n}\right| \log \frac{\left|I_{n}\right|^{1+\alpha-\beta}}{C_{1-\beta}\left(E \cap I_{n}\right)}+\mathscr{I} \log \left(\frac{c}{\mathscr{I}}\|f\|_{\alpha}^{2}\right)=-\infty .
\end{aligned}
$$

By the Fatou Theorem we obtain $f=0$, which finishes the proof.

The following result was obtained by Carleson [4] for the classical Dirichlet space. A generalization of his Theorem was given by Preobrazhenskii in [16] and by Pau and Pelaez in [15] for the Dirichlet spaces $\mathscr{D}_{\alpha}$ with $0<\alpha<1$. Here we give another proof of this generalization.

Corollary 3.2. Let E be a closed subset of $\mathrm{T}$ of Lebesgue measure zero. Let $0<\beta<\alpha \leq 1$. Assume that there exists $m>0$ such that for each interval $I \subset \mathrm{T}$ centered at a point of $E$,

$$
C_{1-\beta}(E \cap I) \geq m|I| .
$$

Then $E$ is a uniqueness set for $\mathscr{D}_{\alpha}$ if and only if

$$
\sum_{n}\left|I_{n}\right| \log \left|I_{n}\right|=-\infty
$$

where $\left(I_{n}\right)_{n}$ are the complementary intervals of $E$.

Proof. Note that $\mathscr{A}^{1}$ (D) $:=\operatorname{Hol}(\mathrm{D}) \cap \mathscr{C}^{1}(\overline{\mathrm{D}}) \subset \mathscr{D}_{\alpha}$. If $E$ is a uniqueness set for $\mathscr{D}_{\alpha}$, then $E$ is a uniqueness set for $\mathscr{A}^{1}$ (D) and thus $E$ is not a Carleson set [4], i.e. $E$ has Lebesgue measure zero and satisfies (10).

Conversely, we write $T \backslash E=\bigcup_{k} I_{k}$ with $I_{k}=\left(e^{i \theta_{2 k}}, e^{i \theta_{2 k+1}}\right)$. Let $J_{2 k}$ (resp. $\left.J_{2 k+1}\right)$ be the open arc of length $\left|I_{k}\right|$ with midpoint $e^{i \theta_{2 k}}\left(\right.$ resp. $\left.e^{i \theta_{2 k+1}}\right)$. By Vitali covering lemma, there exists a sub-collection $\left(J_{k^{\prime}}\right)_{k^{\prime}}$ of $\left(J_{k}\right)_{k}$ which is disjoint and satisfies $\bigcup_{k} J_{k} \subset 3 \bigcup_{k^{\prime}} J_{k^{\prime}}$. Hence,

$$
\sum_{k^{\prime}}\left|J_{k^{\prime}}\right| \log \left|J_{k^{\prime}}\right|=-\infty
$$

Let $F=E \bigcap\left(\bigcup_{k^{\prime}} J_{k^{\prime}}\right)$ be the subset of $E$ contained in $\bigcup_{k^{\prime}} J_{k^{\prime}}$. The set $F$ is a Borel set and, since $F \cap J_{k^{\prime}}=E \cap J_{k^{\prime}}$, by (9),

$$
C_{1-\beta}\left(F \cap J_{k^{\prime}}\right) \geq m\left|J_{k^{\prime}}\right| .
$$


Then for $0<\beta<\alpha \leq 1$, we obtain

$$
\begin{aligned}
\sum_{k^{\prime}}\left|J_{k^{\prime}}\right| \log \frac{\left.\left|J_{k^{\prime}}\right|\right|^{1+\alpha-\beta}}{C_{1-\beta}\left(F \cap J_{k^{\prime}}\right)} & \leq(\alpha-\beta) \sum_{k^{\prime}}\left|J_{k^{\prime}}\right| \log \left|J_{k^{\prime}}\right|-\log m \sum_{k^{\prime}}\left|J_{k^{\prime}}\right| \\
& =-\infty .
\end{aligned}
$$

By Theorem 3.1, the set $F$ is a set of uniqueness for $\mathscr{D}_{\alpha}$ and so does $E$, which finishes the proof.

Remark 3.3. 1. A function $\varphi \in \mathscr{D}_{\alpha}$ is called multiplier of $\mathscr{D}_{\alpha}$ if $\varphi \mathscr{D}_{\alpha} \subset \mathscr{D}_{\alpha}$ and we denote the set of multipliers by $\mathscr{M}_{\mathscr{D}_{\alpha}}$. Richter and Sundberg in [17] proved that a set $E$ is a zero set of Dirichlet space $\mathscr{D}_{1}$ if and only if it is a zero set of $\mathcal{M}_{\mathscr{D}_{1}}$. On the other hand if $\varphi \in \mathscr{M}_{\mathscr{D}_{1}}$, then by Stegenga's result [18] Theorem 2.7.c, we have $\mathscr{D}_{I, 1}(\varphi)=O\left(C_{0}(I)\right)$, note that $C_{0}(I) \asymp|\log I|^{-1}$.

2. Khavin and Maz'ya in [9] have constructed a set of uniqueness $E$ for the classical Dirichlet space such that $C_{1-\beta}(E)=0$ for every $0<\beta<1$. On the other hand, Carleson in [6] has constructed a zero set $E$ which satisfies (10) and $E \cap I$ has a positive logarithmic capacity for all arcs such that $E \cap I \neq \emptyset$. As in [9], we can construct a closed set $E$ which is a set of uniqueness for $\mathscr{D}_{\alpha}$ and such that $C_{1-\beta}(E)=0$ for all $0<\beta<\alpha<1$. Let $\left(l_{n}\right)_{n \geq 0}$ be a sequence in $(0,2 \pi)$ and let $\mathscr{C}$ be the associated generalized Cantor set. Then for $0 \leq s<1$,

$$
C_{s}(\mathscr{C})=0 \Longleftrightarrow \sum_{n} 2^{-n} l_{n}^{-s}=+\infty,
$$

see for example [2], [5].

Choose $l_{n}=\left(2^{-n} n\right)^{\frac{1}{1-\beta}}$. Then $C_{1-\beta}(\mathscr{C})=0$ and for $0<\beta<\alpha$,

$$
\sum_{n} 2^{-n} l_{n}^{-(1-\alpha)}=\sum_{n} 2^{-n \frac{\alpha-\beta}{1-\beta}} n^{-\frac{1-\alpha}{1-\beta}}<\infty
$$

Therefore, $C_{1-\alpha}(\mathscr{C})>0$. Now, consider a family of pairwise disjoint open $\operatorname{arcs}\left(I_{n}\right)_{n}$ of T be such that

$$
\sum_{n}\left|I_{n}\right| \log \left|I_{n}\right|=-\infty
$$

A possible example, $I_{n}=\left(e^{i(\log (n+1))^{-1}}, e^{i(\log n)^{-1}}\right), n \geq 2$. We reproduce the generalized Cantor set $\mathscr{C}$ in each $I_{n}$, which will be denoted by $\mathscr{C}_{n}$. Therefore,

$$
C_{1-\alpha}\left(\mathscr{C}_{n} \cap I_{n}\right) \simeq C_{1-\alpha}(\mathscr{C})\left|I_{n}\right|^{\alpha} .
$$


We set $E=\{1\} \cup \bigcup_{n} \mathscr{C}_{n}$. It is clear that $C_{1-\beta}(E)=0$, for all $0<\beta<\alpha$. Now Theorem 3.1 with $\beta=\alpha$ gives

$$
\begin{aligned}
\sum_{n}\left|I_{n}\right| \log \frac{\left|I_{n}\right|}{C_{1-\alpha}\left(E \cap I_{n}\right)} & \\
& \simeq-\log C_{1-\alpha}(\mathscr{C}) \sum_{n}\left|I_{n}\right|+(1-\alpha) \sum_{n}\left|I_{n}\right| \log \left|I_{n}\right|=-\infty .
\end{aligned}
$$

So $E$ is a set of uniqueness for $\mathscr{D}_{\alpha}$ with $\alpha<1$.

3. Malliavin in [11] gives a complete characterization of the sets of uniqueness for the Dirichlet spaces involving a new notion of capacity, but it appears difficult to apply his result to particular situations (see also [10]).

ACKNOWLEDGMent. I would like to thank the referee for his helpful remarks, specially for those regarding the proof of Theorem 2.2.

\section{REFERENCES}

1. Adams, D., Hedberg, L., Function Spaces and Potential Theory, Grundlehren math. Wiss. 314, Springer, Berlin 1996.

2. Aikawa, H., Essén, M., Potential Theory: Selected Topics, Lecture Notes in Math. 1633, Springer, Berlin 1996.

3. Beurling, A., Ensembles exceptionnels, Acta. Math. 72 (1939), 1-13.

4. Carleson, L., Sets of uniqueness for functions regular in the unit circle, Acta Math. 87 (1952), 325-345.

5. Carleson, L., Selected Problems on Exceptional Sets, Van Nostrand Math. Studies 13, Van Nostrand, Princeton, NJ 1967.

6. Carleson, L., An example concerning analytic functions with finite Dirichlet integrals, Investigations on linear operators and the theory of functions IX, Zap. Nauchn. Sem. Leningrad. Otdel. Mat. Inst. Steklov. (LOMI) 92 (1979), 283-287, 326.

7. Devinatz, A., Hirshman, I., Multiplier transformations on $l^{2, \alpha}$, Ann. of Math. (2) 69 (1959), 575-587.

8. Kahane, J. P., Salem, R., Ensembles parfaits et séries trigonométriques, Actualités Sci. Industr. 1301, Hermann, Paris 1963.

9. Khavin, V., Maz'ya, V., Application of the $(p, l)$-capacity to certain problems of theory of exceptional sets, Math. USSR Sb. 19 (1973), 547-580 (1974).

10. Khavin, V., Khrushchev, S., Sets of uniqueness for analytic functions with the finite Dirichlet integral, Problem 9.3 (pp. 531-535) in: Linear and Complex Analysis Problem Book, Lecture Notes in Math. 1043, Springer, Berlin 1984.

11. Malliavin, P., Sur l'analyse harmonique de certaines classes de séries de Taylor, pp. 71-91 in: Symposia Mathematica XXII, Proc. Roma 1976, Academic Press, London 1977.

12. Maz'ya, V., Conductor and capacitary inequalities for functions on topological spaces and their applications to Sobolev-type imbeddings, J. Funct. Anal. 224 (2005), 408-430.

13. Meyers, N., A theory of capacities for potentials of functions in Lebesgue classes, Math. Scand. 26 (1970), 255-292.

14. Meyers, N., Integral inequalities of PoincaréÄ and Wirtinger type, Arch. Rational Mech. Anal. 68 (1978), 113-120. 
15. Pau, J., Peláez, J. A., On the zeros of functions in Dirichlet spaces, Trans Amer. Math. Soc., 363 (2011), 1981-2002.

16. Preobrazhenskii, S. P., A boundary uniqueness theorem for regular functions with a bounded integral of "Dirichlet type", Zap. Nauchn. Sem. Leningrad. Otdel. Mat. Inst. Steklov. (LOMI) 126 (1983) 180-190.

17. Richter, S., Sundberg, C., Multipliers and invariant subspaces in the Dirichlet space, J. Operator Theory 28 (1992) 167-186.

18. Stegenga, D., Multipliers of the Dirichlet space, Illinois J. Math. 24 (1980), 113-139.

19. Ziemer, W., Weakly Differentiable Functions, Sobolev Space and Functions of Bounded Variation, Grad. Texts in Math. 120, Springer, New York 1989.

UNIVERSITÉ D'AIX-MARSEILLE I

CMI LATP

39 RUE F. JOLIOT-CURIE

13453 MARSEILLE

FRANCE

E-mail: kellay@cmi.univ-mrs.fr 